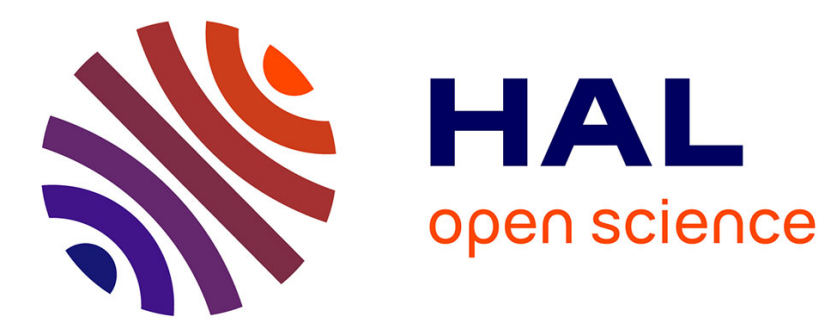

\title{
A priori study for the modeling of LES subgrid scale terms in resolved scale multiphase flows
}

\author{
Mathilde Tavares, Stéphane Vincent, Meryem Ould-Rouiss, Jean-Luc
}

Estivalezes

\section{- To cite this version:}

Mathilde Tavares, Stéphane Vincent, Meryem Ould-Rouiss, Jean-Luc Estivalezes. A priori study for the modeling of LES subgrid scale terms in resolved scale multiphase flows. Notes On Numerical Fluid Mechanics And Multidisciplinary Design, Springer, 2018. hal-01860914

\section{HAL Id: hal-01860914 \\ https://hal.science/hal-01860914}

Submitted on 12 Feb 2020

HAL is a multi-disciplinary open access archive for the deposit and dissemination of scientific research documents, whether they are published or not. The documents may come from teaching and research institutions in France or abroad, or from public or private research centers.
L'archive ouverte pluridisciplinaire HAL, est destinée au dépôt et à la diffusion de documents scientifiques de niveau recherche, publiés ou non, émanant des établissements d'enseignement et de recherche français ou étrangers, des laboratoires publics ou privés. 


\title{
Chapter 1
}

\section{A priori study for the modeling of LES subgrid scale terms in resolved scale multiphase flows}

Mathilde Tavares, Stephane Vincent, Meryem Ould-Rouiss, and Jean-Luc Estivalezes

\begin{abstract}
Modeling accurately the energy transfer across the interface in multiphase flows is difficult. To deal with this phenomenon, the derivation of the governing equations for two-phase flows have been formulated. A priori tests are used in order to evaluate the relative magnitude of unclosed LES specific to multiphase flows. There consist in the explicit filtering of 3D Direct Numerical Simulation in order to find LES models appropriated to the different subgrid contributions. In our study, explicit volume filtering and phase weighted filtering have been used in a case of phase separation flow in a cubic closed box between water, the heavier fluid and oil, the lighter fluid, in order to understand the effect of the filtering process on the subgrid contributions
\end{abstract}

\subsection{Introduction}

Two-phase flows are involved in many industrial and environmental applications such as spray formation, wave breaking or oil transportation. The modeling and simulation of complex interactions between turbulence and interface in multiphase flows remain challenging. As in single phase flow, large Eddy Simulation (LES) solve the problem of large scale while smaller subgrid scales are computed through physical or mathematical models in two-phase flows, involving this way several unclosed subgrid terms characteristic of turbulent and interfacial motion subgrid correlations. Several works [5], [3], have established a hierarchy of unclosed subgrid terms through a priori studies to improve LES simulation of two-phase flows. The

Mathilde Tavares Stephane Vincent · Meryem Ould-Rouiss ·

Paris Est Marne La Vallée University, France, e-mail: mathilde.tavares@u-pem.fr

Jean-Luc Estivalezes

Onera The french aerospace lab, France 
main objective of the present work is to characterize the hierarchy of subgrid terms for two-phase flows in the case of a phase inversion flow. In the following sections the 1-fluid model is presented and filtered to highlight the unclosed LES terms for two-phase flows. Then, we present the reference simulation, a phase separation flow from a numerical benchmark [6]. Finally the results of a priori tests are discussed to evaluate the relative importance of LES subgrid terms in the reference case.

\subsection{Numerical modeling}

\subsubsection{Navier Stokes equations/1-fluid model for two-phase flows}

The two-phase flow numerical modeling is restricted to immiscible, incompressible and isothermal fluids. For two non miscible fluid where an index $k$ refers to one phase if $k=1$ and the other phase if $k=2$, the dynamic of each phase $k$ is governed by the Navier-Stokes equations. Thanks to jump relations, mass and momentum conservation is ensured at the interface. By adding these boundary conditions to NavierStokes equations, it is possible to build in the framework of the 1-fluid formalism of [2] a unique set of equation valid in both phase by introducing the volume fraction function $C$ which is equal to 1 in one phase an 0 elsewhere and describes the interface evolution through a material advection equation (1.1).

$$
\begin{aligned}
\nabla \cdot \mathbf{u} & =0 \\
\frac{\partial \rho \mathbf{u}}{\partial t}+\nabla \cdot(\rho \mathbf{u} \otimes \mathbf{u}) & =-\nabla P+\rho \mathbf{g}+\nabla \cdot(2 \mu \mathbf{S})+\sigma \kappa \mathbf{n}_{i} \delta_{i} \\
\frac{\partial C}{\partial t}+\mathbf{u} \cdot \nabla C & =0
\end{aligned}
$$

$\mathbf{u}$ is the velocity, $P$ the pressure, $t$ the time, $\mathbf{g}$ the gravity vector, $\rho$ the density, $\mu$ the dynamic viscosity. $\mathbf{S}_{k}=\frac{1}{2}\left(\nabla \mathbf{u}_{k}+\nabla^{T} \mathbf{u}_{k}\right)$ is the viscous stress tensor. $\sigma \kappa \mathbf{n}_{i}$ is the surface tension force where $\sigma$ denotes the surface tension coefficient, $\kappa$ the local curvature and $\mathbf{n}_{i}$ the normal to the interface.

Local quantities such as density $\rho$ or viscosity $\mu$ can be defined by using the volume fraction function $C$.

$$
\begin{aligned}
& \rho=\rho_{2}+(1-C) \rho_{1} \\
& \mu=\mu_{2}+(1-C) \mu_{1}
\end{aligned}
$$

The curvature $\kappa$ depends on the interface topology. It can modeled using the Continuum Surface formulation of [1]:

$$
\kappa \mathbf{n}_{i} \delta_{i}=\nabla \cdot\left(\frac{\nabla C}{\|\nabla C\|}\right) \nabla C, \mathbf{n}=\frac{\nabla C}{\|\nabla C\|}, \mathbf{n}_{i} \delta_{i}=\nabla C
$$




\subsubsection{Filtered Navier-Stokes equations for two-phase flow}

LES formalism required the application of a low-pass frequency filtering to the field to resolve large turbulent scales while the effects of smaller scales are modeled [4]. The filtering operation of a hydrodynamic field is defined on a domain $\Omega$ by:

$$
\overline{\Phi(x, t)}=G * \Phi \rightarrow \overline{\Phi(x, t)}=\int_{\Omega} \int_{-\infty}^{t} G\left(\bar{\Delta}(x, t), x-x^{\prime}, t-t^{\prime}\right) \Phi\left(x^{\prime}, t^{\prime}\right) d x^{\prime} d t^{\prime}
$$

$\bar{\Delta}$ is the cutoff lengthscale of the filter, $G$ a formal operator. It can be assumed that the filtering operator commutes with time and spatial derivatives. Then, the volume filtering of incompressible Navier-Stokes equations depend on $\{\overline{\mathbf{u}}, \bar{P}, \bar{C}, \bar{\rho}, \bar{\mu}, \hat{\kappa}\}$ and can be written as:

$$
\begin{aligned}
& \nabla \cdot \overline{\mathbf{u}}=\quad 0 \\
& \frac{\partial \bar{\rho} \overline{\mathbf{u}}}{\partial t}+\nabla \cdot(\bar{\rho} \overline{\mathbf{u}} \otimes \overline{\mathbf{u}})-\bar{\rho} \mathbf{g}+\nabla \bar{P}-\nabla \cdot(2 \bar{\mu} \overline{\mathbf{S}})-\sigma \hat{\kappa} \nabla \bar{C}=-\nabla \cdot\left(\tau_{c o n v}-\tau_{\text {diff }}\right)+ \\
& \tau_{\text {superf }}-\frac{\partial \tau_{\text {temps }}}{\partial t} \\
& \frac{\partial \bar{C}}{\partial t}+\overline{\mathbf{u}} \cdot \nabla \bar{C}=\quad \tau_{\text {interf }}
\end{aligned}
$$

This approach is divergence free but required modeling of 5 subgrid terms. It can be more convenient to use the Favre average or mass weighted filtering for the velocity field such as

$$
\tilde{\mathbf{u}}=\frac{\overline{\rho \mathbf{u}}}{\bar{\rho}}
$$

This approach is based on $\{\tilde{\mathbf{u}}, \bar{P}, \bar{C}, \bar{\rho}, \bar{\mu}, \hat{\kappa}\}$. With this formulation, the velocity field is no more divergence free with the additional source term $\frac{\tau_{\text {interf }}\left(\rho_{2}-\rho_{1}\right)}{\bar{\rho}}$ but only 4 subgrid terms require modeling. A summary of the subgrid term for the classic volume filtering and the phase weighted filtering are given in the table (1.1).

\begin{tabular}{c|c|c}
\hline Subgrid terms & Volume filter & Mass weighted filter (Favre) \\
\hline$\partial \tau_{\text {temps }} / \partial t$ & $\frac{\partial \rho \mathbf{u}}{\partial t}-\frac{\partial \bar{\rho} \overline{\mathbf{u}}}{\partial t}$ & $\left(\rho_{2}-\rho_{1}\right) \tilde{\mathbf{u}} \tau_{\text {interf }}$ \\
\hline$\nabla \cdot \tau_{\text {conv }}$ & $\nabla \cdot(\overline{\rho \mathbf{u} \otimes \mathbf{u}}-\bar{\rho} \overline{\mathbf{u}} \otimes \overline{\mathbf{u}})$ & $\nabla \cdot(\bar{\rho}(\overline{\mathbf{u} \otimes \mathbf{u}-\tilde{\mathbf{u}} \otimes \tilde{\mathbf{u}}))}$ \\
\hline$\nabla \cdot \tau_{\text {diff }}$ & $\nabla \cdot(\overline{2 \mu \mathbf{S}}-2 \mu \overline{\mathbf{S}})$ & $\nabla \cdot(\overline{2 \mu \mathbf{S}}-2 \bar{\mu} \mathbf{S})$ \\
\hline$\tau_{\text {superf }}$ & $\sigma(\overline{\kappa \nabla C}-\hat{\kappa} \nabla \bar{C})$ & $\sigma(\overline{\kappa \nabla C}-\hat{\kappa} \nabla \bar{C})$ \\
\hline$\tau_{\text {interf }}$ & $\overline{\mathbf{u} . \nabla C}-\overline{\mathbf{u}} . \nabla \bar{C}$ & $\overline{\mathbf{u} . \nabla C}-\tilde{\mathbf{u}} \nabla \bar{C}$ \\
\hline
\end{tabular}

Table 1.1: Subgrid terms for two-phase flow 


\subsection{A priori filtering of phase separation flow}

\subsubsection{Description of the problem}

Our study is based on the numerical benchmark of [6] where a parametric study on macroscopic quantities such as kinetic or potential energy have been performed with different codes and meshes to characterize vorticity generation on a phase separation flow between water and oil (1.1a). We have chosen to deal only with the turbulent

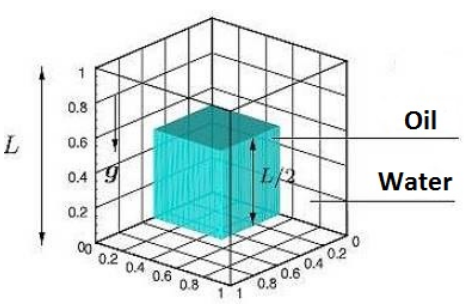

(a) Initial conditions

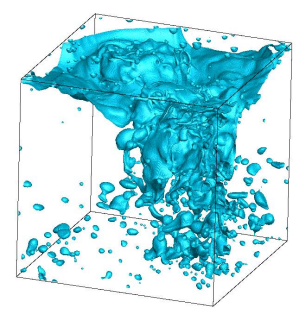

(b) Enstrophy peak

Fig. 1.1: Initial conditions of phase inversion flow problem (1.1a) and DNS solution at enstrophy peak (1.1b)

case where the properties are given in the table (1.2) below. We have post-processed

\begin{tabular}{c|c|c|c|c|c|c|c|c}
\hline & $\rho\left(\mathrm{kg} / \mathrm{m}^{3}\right)$ & $\mu($ Pa.s $)$ & $\sigma(N . m)$ & $R e$ & $W e$ & $L(m)$ & $\eta(\mathrm{m})$ & $D_{g}(\mathrm{~m})$ \\
\hline Oil & 900 & 0,1 & 0,45 & 221472 & 109 & 1 & $4,515.10^{-4}$ & $9,174.10^{-3}$ \\
\hline Water & 1000 & 0,001 & - & - & - & - & - & - \\
\hline
\end{tabular}

Table 1.2: Fluids characteristic for the phase inversion problem

results of DyJeat (Dynamic of Jet ATomization), a computational in-house code developed at ONERA/DMAE. DyJeat is implemented with the finite volume method for the discretization of Navier-Stokes equations on a staggered mesh.The GhostFluid method is used to deal with surface tension forces, density and viscosity jumps at the interface. The Level-Set approach is also used for tracking interfaces and a Level-Set/VOF coupling is ensured to improve mass conservation. Details of implementation and validation can be found in [6]. All the fields have been taken for a single time at the enstrophy peak $(1.1 \mathrm{~b})$ corresponding to the time where the turbulence is maximum. Three meshes $\left(128^{3}, 256^{3}, 512^{3}\right)$ have been used in order to study the effect of the mesh on the subgrid contributions. Moreover, we have used two different filter size, G2 and G4, and two filter's type, the volume filtering and the mass weighted filtering to evaluate the influence of the type and the size of the 
filter. The size of the filter $\mathrm{G} 2$ is for instance:

$$
G^{(2)}\left(\Phi_{i j k}\right)=\int_{x_{i-1}}^{x_{i+1}} \int_{y_{j-1}}^{y_{j+1}} \int_{z_{k-1}}^{z_{k+1}} \Phi(x, y, z) d x d y d z
$$

For each subgrid term, a spatial sum have been computed in each direction and compared to the spatial sum of the resolved convective term $\nabla .(\bar{\rho} \overline{\mathbf{u}} \otimes \overline{\mathbf{u}})$ for the momentum equation or the resolved interface advection term $\overline{\mathbf{u}} . \nabla \bar{C}$ for the advection equation to show the order of magnitude of subgrid contributions. The figures (1.2a)

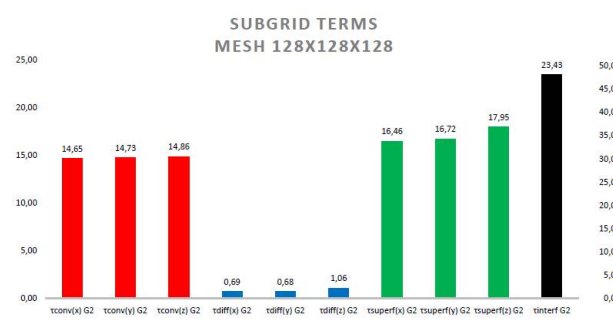

(a) $128^{3}$

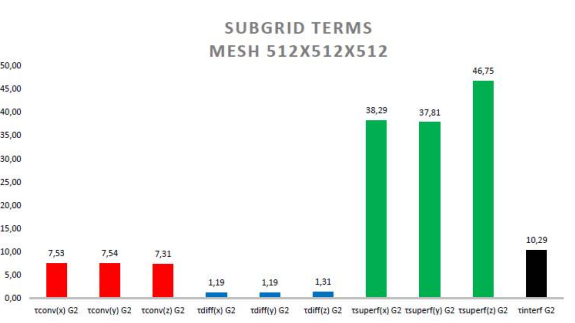

(b) $512^{3}$

Fig. 1.2: Subgrid term hierarchy for meshes $128^{3}$ (1.2a) and $512^{3}$ (1.2b) with volume filtering filter along $x, y, z$ directions

and (1.2b) shows the order of magnitude of subgrid contributions for the meshes $128^{3}$ and $512^{3}$ in each direction. Generally, the highest value of the subgrid contributions is along the $z$ direction which due to anisotropy of the phase inversion flow. In agreement with [3], [5], our a priori analysis shows that the subgrid viscous term $\tau_{\text {diff }}$ is always negligible. Besides, $\tau_{\text {superf }}$, the subgrid contribution linked to the surface tension, is the dominant term. Indeed, capillarity drives the phase inversion mechanism at the enstrophy peak with important rupture and coalescence phenomena, increasing the contribution of $\tau_{\text {superf }}$. The convective $\tau_{\text {conv }}$ and interfacial $\tau_{\text {interf }}$ subgrid terms have the same order of magnitude but remain significant. We observe a strong influence of the mesh on unclosed LES terms. Indeed, all the subgrid contributions decrease when the mesh size becomes smaller except for $\tau_{\text {superf }}$. On the other hand, the figure (1.3b) shows that subgrid contributions are bigger when we use volume filtering than phase weighted average as shown in literature. According to [3], using volume filtering process overestimates the convective transfer across the interface whereas phase weighted underestimates it. The figure (1.3a) shows that the filter size (G2 and G4) has almost the same effect than the mesh size on the subgrid contributions and for the smaller filter size, unclosed LES terms are weaker even for $\tau_{\text {superf }}$ in our case. 




(a) Comparison filter G2 and G4

(b) Comparison volume and phase weighted filter

Fig. 1.3: Subgrid term hierarchy for meshes $512^{3}$ depending on the size of filter (1.3a) and the type of the filter (1.3b) along $z$ direction

\subsection{Conclusion}

In this study, we have shown that the contribution linked to the surface tension has to be taken into consideration. In the case of the phase inversion flow, at the enstrophy peak, $\tau_{\text {superf }}$ and $\tau_{\text {interf }}$ has been the predominant term, whatever the mesh considered. It's worth noting that the mesh size here is four times smaller than those studied in literature whereas the Weber is six times smaller. The capillarity effects are then more important and better resolved with our finer grid, increasing the part of the capillary subgrid term. To conclude, this term cannot be neglected and it is then necessary to model it properly to improve LES multiphase flow simulation, in addition to inertial terms. It has to be noted that the $\tau_{\text {interf }}$ is also not negligible and can lead to non divergence free filtered LES fields.

\section{References}

1. J.U Brackbill, D.B Kothe, and C Zemach. A continuum method for modeling surface tension. Journal of Computational Physics, 100(2):335-354, 1992.

2. I. Kataoka. Local instant formulation of two-phase flow. International Journal of Multiphase Flow, 12(5):745-758, 1986.

3. E. Labourasse, D. Lacanette, A. Toutant, P. Lubin, S. Vincent, O. Lebaigue, J.-P. Caltagirone, and P. Sagaut. Towards large eddy simulation of isothermal two-phase flows: Governing equations and a priori tests. International journal of multiphase flow, 33:1-39, 2007.

4. P. Sagaut. Large Eddy Simulation for Incompressible Flows. Springer, 1998.

5. S. Vincent, J. Larocque, D. Lacanette, A. Toutant, P. Lubin, and P. Sagaut. Numerical simulation of phase separation and a priori two-phase les filtering. Computers and Fluid, 37:898-906, 2008.

6. S. Vincent, L. Osmar, J.-L. Estivalezes, S. Zaleski, F. Auguste, W. Aniszewski, Y. Ling, T. Menard, A. Pedrono, J. Magnaudet, J.-P. Caltagirone, and A. Berlemont. A phase inversion benchmark for multiscale multiphase flows. Journal of Computational Physics, Submitted, 2015. 